\title{
Papillary Carcinoma of the Breast Among Omani Women: A Case Series and a Literature Review
}

\author{
Bahaaeldin Baraka*, Al Kharusi Suad, Al Farsi Abdulaziz \\ Department of Medical Oncology, National Oncology Centre, Royal Hospital, Muscat, Oman \\ Email address: \\ Bahaabaraka@gmail.com (B. Baraka) \\ ${ }^{*}$ Corresponding author \\ To cite this article: \\ Bahaaeldin Baraka, Al Kharusi Suad, Al Farsi Abdulaziz. Papillary Carcinoma of the Breast Among Omani Women: A Case Series and a \\ Literature Review. Journal of Cancer Treatment and Research. Vol. 7, No. 3, 2019, pp. 47-50. doi: 10.11648/j.jctr.20190703.11
}

Received: August 21, 2019; Accepted: September 6, 2019; Published: October 10, 2019

\begin{abstract}
Breast cancer is the most common cause of death from cancer in women worldwide. In 2012, an estimated of 100,000 cases of invasive breast cancer were diagnosed in the United States. The histopathology type is predominantly ductal in about $70-80 \%$ of patients followed by invasive lobular carcinoma in $5-15 \%$ of case. Invasive papillary carcinomas of the breast are rare, accounting for less than $1-2 \%$ of invasive breast cancers. Occasionally the disease is found in men as well. Papillary carcinoma most frequently occurs in older and post-menopausal women. Triple assessment is essential to reach the diagnosis. The typical sonographic appearance of IPC is of hypoechoic area with soft tissue echoes emerging from the wall of the cyst. Invasive micropapillary carcinoma is a luminal-type breast cancer with a propensity for lymphovascular invasion and regional lymph-node metastasis. Intracystic papillary breast cancer (IPC) is best managed in the context of a multidisciplinary team. Surgical excision of the lump with margins in excess of $2 \mathrm{~mm}$ is considered satisfactory. Many studies suggest that papillary carcinoma may have a better prognosis than invasive ductal carcinoma (IDC). However, the little available information about papillary carcinoma of the breast underscores the need for further management related studies.
\end{abstract}

Keywords: Breast, Cancer, Papillary, Metastatic, Chemotherapy

\section{Introduction}

According to the World Health Organization (WHO), Invasive papillary carcinoma (IPC) of the breast is a uniquely differentiated adenocarcinoma with a papillary morphology, but otherwise has no clinical, genetic, or prognostic special features [1]. The diagnosis of invasive carcinoma can only be made if invasion is present outside the surrounding fibrous capsule. If not, encapsulated papillary carcinoma should be staged as papillary carcinoma in situ [2]. Histologically, they are characterized by different scales of the finger-like protrusions or leaf-like structure and the presence of arborescent fibrovascular cores covered with myoepithelial cell. Normal breast gland tissue does not show papillary morphology, and related studies on the mechanism of papillary appearance are rare [3].

Invasive papillary carcinomas of the breast typically present with bloody discharge per nipple, a breast mass, or mammographic abnormalities. [4]. Immunohistochemical analyses of papillary carcinoma suggest the utility of markers such as smooth muscle myosin heavy chain, calponin, p63, and high molecular weight keratins, which can characterize the myoepithelial cell layer [5]. The assessment and categorization of papillary lesions remains one of the most challenging areas in breast pathology [6]. Histologically, there are proliferations of cells arranged around fibrovascular cores, usually grossly forming a mass with a well-defined border and is made up of small, finger-like projections [7].

Triple assessment is mandatory to reach the diagnosis. The mammographic appearance of invasive papillary carcinoma is not always specific and may often range from mammographically negative small papillary carcinomas to a well-circumscribed dense mass, causing a minimal to moderate duct dilatation in a tapering density pattern from the nipple toward the parenchyma, [8] meanwhile the typical sonographic appearance of IPC is of hypoechoic area with soft tissue echoes emerging from the wall of the cyst.

Fine-needle aspiration cytology or a core biopsy is usually the first diagnostic tool. Aspirates from papillary carcinomas are more often characterized by high cellularity, prominent 
papillary or cribriform configuration, many single columnar epithelial cells, with haemorrhage and hemosiderin-laden macrophages in the background. The majority of these tumours are low grade, hormone-receptor positive, and show an overall favourable prognosis. [9]

A total mastectomy, modified radical mastectomy, segmentectomy either with or without sentinel node biopsy, adjuvant hormonal therapy or chemotherapy, and radiotherapy, all alone or in combination, have been contemplated as treatment options. [1]

Herein, we are reporting here four cases of IPC and do a brief review of the literature on this topic.

\section{Materials and Methods}

\subsection{Materials}

All Invasive papillary carcinomas of the breast patients who were treated at the Royal Hospital from January 2014 to June 2018 were included in the study. Electronic medical records were reviewed.

\subsection{The Studies Parameters}

The clinicopathological features and treatment outcomes of breast cancer patients with Invasive papillary carcinoma were studied. Clinicopathological features including age, pathological type and grade, prognostic and predictive markers, stage at the time of diagnosis, the presenting symptoms, type of treatment before and after surgery were recorded and analyzed.

\subsection{The Cases}

a. Case 1

A 73-year-old lady, is known to have hypertension and type 2 diabetes mellitus and she uses oral hypoglycemic medications. Presented in April 2017 with left breast lump, for which she had mammogram and breast ultrasound that revealed two adjacent complex cystic lesions were seen in retro-areolar area, the lesions had fluid levels with intracystic hypervascular and solid components, one of them measured $3.3 \times 3.2 \mathrm{~cm}$ and its solid component was measuring $1.0 \times 0.6$ $\mathrm{cm}$, the other lesion was measuring $3.5 \times 3.2 \mathrm{~cm}$ and its solid component was measuring $2.7 \times 1.5 \mathrm{~cm}$ in size; together measuring $6.8 \times 3.3 \mathrm{~cm}$ in size. Both the skin and nipple were unremarkable. Therefore she underwent left breast whole lump excision along with sentile lymph node dissection (SLND). Histopathologic examination showed intracystic (encapsulated) papillary carcinomas with adjacent small solid papillary carcinoma ( $\mathrm{pT} 1)$. The solid papillary carcinoma was strongly positive for both estrogen (ER) and progesterone receptors (PR). The case was discussed in multi-disciplinary team meeting and the plan was to treat the patient as invasive carcinoma; however, in view of her age, multiple co-morbidities, the early stage lesion and receptors' positive status post operative radiation was deferred and patient commenced on endocrine therapy in the form of aromatse inhibitors (Letrazole). The patient has been followed up for the last 15 months and has no evidence of recurrence.

b. Case 2

A 48-year-old premenopausal female patient, married and has 6 children all of them had been breastfed. Presented with an 8-month history of bloody discharge per her right nipple and feeling of right breast lump. Breast ultrasound and mammogram revealed multiple dilated ducts, seen at 11 o'clock, some showed clear contents, other at 12 o'clock showed soft tissue echogencity as nodules along the duct, in addition to large intraductal masses extending outside the duct, measuring $14 \times 12$ and $9 \times 5 \mathrm{~mm}$, with high vascularity, resembling a picture of intraductal papillomatosis, BIRAD 4. Ultrasound guided biopsy was performed for her and histological examination didn't reveal any intraductal papillary lesions, however it showed blood vessels ectasia, which suggested a benign vascular lesion. She underwent breast lump excision and histological examination revealed an encapsulated papillary carcinoma, less than $1 \mathrm{~mm}$ from the inked margins, staged as carcinoma in-situ (Tis) disease. ER and PR were positive, KI67 Proliferative index was 15\% while human epidermal growth factor receptor 2 (HER2) was negative. The patient received adjuvant conventional radiotherapy after the breast conserving surgery with the standard three-dimensional conformal radiotherapy (3DCRT), followed by adjuvant hormonal therapy (Tamoxifen). At his last follow up, more than two years after her initial diagnosis, the patient remained free of breast cancer recurrence.

c. Case 3

A 75-year-old man, presented with a left breast slowly growing mass. He reported no family history of breast or ovarian cancer, although his own medical history included hypertension, dyslipidemia and JAK-2 Positive myeloproliferative disorder (polycthemia vera) used to be treated with hydroxyurea and aspirin. A bilateral mammogram and left breast ultrasound showed an $8 \times 8 \mathrm{~cm}$ cystic lesion in favour of epidermal inclusion cyst. He underwent a left lumpectomy. Histological examination revealed fibro-collagenous cyst wall with protrusion of complex papillary structures in the lumen, lined by mildly to moderately pleomorphic epithelial cells and scattered solid tumor nests and foci of calcification within the wall with focal extension to the outer inked cyst wall margins without Lympho-vascular invasion, favouring of encapsulated invasive papillary carcinoma grade 3 . Therafter, he had completion left mastectomy and axillary lymph node dissection, revealed fat necrosis, foreign body granulomas, and fibrosis, with no evidence of residual previous pathology (encysted papillary carcinoma) or other malignancy, all margins were negative for carcinoma in situ or invasive malignancy; moreover, no evidence of malignancy in the excised 18 lymph nodes. Immunohistochemistry showed positive both estrogen (ER) and progesterone receptors (PR) and negative HER-2. Staging investigations including a bone scan, abdominal ultrasound, and a chest x-ray showed no evidence of metastatic disease. Post-operative bilateral 
mammography revealed left breast post surgical changes and interestingly right gynecomastia. The patient commenced on adjuvant endocrine therapy (Tamoxifen) and at his most recent follow up, more than 11 months after his initial diagnosis, the patient showed no signs of recurrence.

d. Case 4

A 75-year-old lady, presented with right breast lump for 3 months. Patient has history of right breast fibrocyctic disease 4 years ago; moreover, she has been suffering from hypertension which has been treated with Atenolol $50 \mathrm{mg}$ daily. A bilateral mammogram and left breast ultrasound showed a right breast cystic lesion at 11 o'clock 42.4 X 2.9 mm. BIRADS 4. She underwent a right breast lumpectomy. Histological examination revealed intracystic encapsulated papillary carcinoma with low grade solid and cribriform DCIS, no capsular invasion and margins were unremarkable. The solid atypical proliferation was diffusely and strongly positive for estrogen and progesterone receptors. HER2 immunostain was equivocal (score $2+$ ). Postoperatively, the patient commenced on endocrine therapy (Tamoxifen) and at her most recent follow up, approximately 3 months after her initial diagnosis, the patient was well, and showed no signs of recurrence.

\section{Discussion}

This report aimed to shed light on this rare form of breast cancer, and to describe the presentation, management, and outcome of four cases of invasive papillary carcinomas of the breast who presented to our centre with either a palpable mass or discharge from the nipple. Each of the above patients had been managed in the context of a multidisciplinary team according to the most common treatments for male breast cancer $(\mathrm{MBC})$ and female breast cancer (FBC). Till date none of the patients experienced local recurrence of their breast cancer.

In the context of pathology, the term papillary carcinoma entails a morphologically heterogeneous group of lesions have a growth pattern characterized by the presence of fibrovascular stalks lined by epithelial cells. Breast papillary neoplasm encompasses a different number of microscopically distinct lesions including ductal carcinoma in situ (DCIS) arising in an intraductal papilloma, papillary DCIS, enscapsulated papillary carcinoma, solid papillary carcinoma, and invasive papillary carcinoma [7,10].

Genetic features may also help to in distinguish papillary carcinoma from other benign lesions. Microsatellite markers of papillary carcinoma specimens assessment suggested that loss of heterozygosity ( $\mathrm{LOH}$ ) of $16 \mathrm{q} 23$ was specific to malignant lesions. [11]

Surgical excision of the breast lump with margins of $\geq 2$ $\mathrm{mm}$ is considered satisfactory. The available data recommend sentinel lymph node biopsy (SLNB) because of the possibility of the presence of invasive cancer in the final histology. Radiation therapy following breast invasive papillary carcinomas alone is of uncertain significance as this form of cancer is usually low grade and rarely recurs.
Nonetheless, in young (less than 50 years old) patients with pure IPC and associated DCIS or micro-invasion, there is some evidence to suggest that adjuvant radiation and endocrine therapy should be considered to reduce the risk of local recurrence [6].

This series describes the cases of three women and one man, diagnosed with some form of papillary carcinoma, regarding the late, there is currently no standard protocol for diagnostic imaging in male breast patients; investigation is typically based on the treatment of female patients [12]. The rarity of male breast cancer amount to a challenge, and management methods that are optimized for men should be further investigated, in order to be treated optimally. Intracystic papillary carcinoma of the male breast represents an extremely rare entity that accounts for less than $1 \%$ of all malignancies, and histologically it ranges from papillary hyperplasia in gynecomastia to invasive papillary carcinoma. [1]

In comparison to invasive ductal carcinoma, papillary carcinoma has a relatively higher survival rate and lower rate of recurrence, although other studies have not found a significant difference between the two forms of breast cancer $[2,5,9]$.

\section{Conclusion}

Further research is required to understand the low-grade nature of this unique form of breast cancer and to determine the role of adjuvant radiotherapy and endocrine therapy. More data analysis and larger samples size are needed to define the appropriate treatment and allow treatment options to be less radical and safely omitted. Given that the overall good prognoses and that there is no adjuvant treatment which increases cancer-free survival, there is a concern for potential overtreatment in this disease [13].

\section{References}

[1] Blaumeiser B, Tjalma WA, Verslegers I, et al. (2002) Invasive papillary carcinoma of the male breast. Eur Radiol 12: 2207-2210.

[2] Sinn HP, Kreipe H (2013) A Brief Overview of the WHO Classification of Breast Tumors, $4^{\text {th }}$ Edition, Focusing on Issues and Updates from the $3^{\text {rd }}$ Edition. Breast Care (Basel) 8: $149-154$.

[3] Rakha EA. Morphogenesis of the papillary le-sions of the breast: phenotypic observation. J Clin Pathol 2016; 69: 64-69.

[4] Reefy SA, Kameshki R, Sada DA, et al. (2013) "Intracystic papillary breast cancer: a clinical update". Ecancermedicalscience 7: 286.

[5] Pal SK, Lau SK, Kruper L, et al. (2011) Papillary Carcinoma of the Breast: An Overview. Breast Cancer Res Treat 122: 637-645.

[6] Collins LC, Schnitt SJ. Papillary lesions of the breast: selected diagnostic and management issues. Histopathology. 2008 Jan; 52 (1): 20-9. [PubMed] 
[7] Ueng SH, Mezzetti T, Tavassoli FA. Papillary neoplasms of the breast: a review. Arch Pathol Lab Med. 2009 Jun; 133 (6): 893-907.

[8] Levine PH, Waisman J, Yang GC. Aspiration cytology of cystic carcinoma of the breast. Diagn Cytopathol 2003; 28: 39-44.

[9] Clement Z, Jones M (2017) Solid Papillary Carcinoma of the Breast: A Review. Int J Surg Med 3: 57-59.

[10] Mulligan AM, O'Malley FP. Papillary lesions of the breast: a review. Adv Anat Pathol. 2007 Mar; 14 (2): 108-19.
[11] Cristofano CD, Mrad K, Zavaglia K, Bertacca G, Aretini P, Cipollini G, et al. Papillary lesions of the breast: a molecular progression? Breast Cancer Research and Treatment. 2005; 90 (1): 71-6.

[12] Grabowski J, Salzstein SL, Sadler GR, Blair S. Intracystic papillary carcinoma: A review of 917 cases. Cancer 2008; 113: 916-20. [PUBMED]

[13] Iuanow E, Kettler M, Slanetz PJ (2011) Spectrum of disease in the male breast. AJR Am J Roentgenol 196: 247-259. 